\title{
Predictive factors for new onset or progression of knee osteoarthritis one year after trauma: MRI follow-up in general practice
}

\author{
Ingrid M. Koster • Edwin H. G. Oei • Jan-Hein J. Hensen • Simone S. Boks • \\ Bart W. Koes • Dammis Vroegindeweij • M. G. Myriam Hunink •
}

Sita M. A. Bierma-Zeinstra

Received: 14 October 2010 /Revised: 19 December 2010 / Accepted: 16 January 2011 /Published online: 6 March 2011

(C) The Author(s) 2011. This article is published with open access at Springerlink.com

\begin{abstract}
Objective To prospectively evaluate prognostic factors for new onset or progression of degenerative change on followup MRI one year after knee trauma and the association with clinical outcome.
\end{abstract}

I. M. Koster · J.-H. J. Hensen · S. S. Boks · D. Vroegindeweij

Department of Radiology, Maasstad Ziekenhuis,

Postbus 9100, 3007 AC Rotterdam, The Netherlands

\section{E. H. G. Oei • M. G. M. Hunink}

Program for the Assessment of Radiological Technology (ART

Program), Erasmus MC,

University Medical Center,

Rotterdam, The Netherlands

E. H. G. Oei • M. G. M. Hunink $(\bowtie)$

Department of Radiology, Erasmus MC,

University Medical Center,

Dr. Molewaterplein 50,

3015 GE Rotterdam, The Netherlands

e-mail: m.hunink@erasmusmc.nl

E. H. G. Oei $\cdot$ S. S. Boks $\cdot$ M. G. M. Hunink

Department of Epidemiology, Erasmus MC,

University Medical Center Rotterdam,

Dr. Molewaterplein 50,

3015 GE Rotterdam, The Netherlands

B. W. Koes $\cdot$ S. M. A. Bierma-Zeinstra

Department of General Practice, Erasmus MC,

University Medical Center,

Rotterdam, The Netherlands

Present Address:

J.-H. J. Hensen • D. Vroegindeweij

Department of Radiology, Maasstad Ziekenhuis,

Postbus 9100, 3007 AC Rotterdam, The Netherlands
Methods Within a prospective observational cohort study in general practice, we studied a subgroup of 117 patients with acute knee trauma (mean age 41 years, $43 \%$ women). Degenerative change was scored on MRI at baseline and after one year follow-up. Multivariate logistic regression

Present Address:

I. M. Koster

Department of Radiology, Erasmus MC,

University Medical Center,

Dr. Molewaterplein 50,

3015 GE Rotterdam, The Netherlands

Present Address:

E. H. G. Oei

Department of Radiology, Erasmus MC,

University Medical Center Rotterdam,

Dr. Molewaterplein 50,

3015 GE Rotterdam, The Netherlands

Present Address:

S. S. Boks

Department of Radiology,

Diaconessenhuis Meppel, Hoogeveenseweg 38,

7943 KA Meppel, The Netherlands

Present Address:

B. W. Koes - S. M. A. Bierma-Zeinstra

Department of General Practice, Erasmus MC,

University Medical Center Rotterdam,

Dr. Molewaterplein 50,

3015 GE Rotterdam, The Netherlands 
analysis was performed to evaluate prognostic factors for new onset or progressive degenerative change on follow-up MRI. Association between new or progressive degeneration and clinical outcome after one year was assessed.

Results On follow-up MRI 15\% of patients with pre-existing knee osteoarthritis showed progression and $26 \%$ of patients demonstrated new degenerative change. The only statistically significant prognostic variable in the multivariate analysis was bone marrow oedema on initial MRI (OR 5.29 (95\% CI 1.6417.1), $p=0.005$ ). A significant association between new or progressive degenerative change and clinical outcome was found $(p=0.003)$.

Conclusion Bone marrow oedema on MRI for acute knee injury is strongly predictive of new onset or progression of degenerative change of the femorotibial joint on follow-up MRI one year after trauma, which is reflected in clinical outcome.

Keywords Knee injury · Osteoarthritis · MRI · Follow-up · Clinical outcome

\section{Introduction}

Osteoarthritis (OA) of the knee is a common cause of functional impairment and pain in the general population. Although the pathogenesis and aetiology of OA has not been fully understood, it is regarded as a generalised degenerative process involving all tissues of the affected joint [1]. Besides demographic factors such as age and sex, obesity and knee trauma are established risk factors for the development of OA of the knee [2-4]. With a reported incidence of 5.3 per 1000 patients annually, traumatic knee injury is a frequently encountered condition in general practice [5].

Meniscal lesions and anterior cruciate ligament (ACL) injuries constitute a large proportion of traumatic knee injury, and evidence exists that traumatic meniscal lesions are associated with progression of knee osteoarthritis [6, 7]. ACL injuries most commonly occur in young- to middle-aged physically active people, and in a previous study it was found that approximately $50 \%$ of this population develop OA of the knee 10-20 years after the injury [8]. It has also been suggested that bone marrow oedema is a risk factor for structural deterioration and progression of knee OA $[9,10]$.

Detection of early OA changes in the knee by radiographs alone is limited and the correlation between radiographic findings and clinical symptoms is poor [11-13]. With magnetic resonance imaging (MRI) it is possible to separately evaluate bone, cartilage, ligaments, meniscus and soft tissue abnormalities, allowing an in-depth whole organ assessment rather than an indirect radiographic projection of intra-articular damage. Therefore, MRI has been advocated as the best currently available imaging technique for the detection of early osteoarthritic changes $[6,14,15]$.

The extent to which traumatic knee abnormalities predict the development of OA within the first years after trauma has only been sparsely documented. We performed a study in the general practice population to prospectively evaluate prognostic factors, including initial MRI findings, for new onset or progression of $\mathrm{OA}$ of the femorotibial joint, assessed by follow-up MRI one year after trauma, and to evaluate the association with clinical outcome.

\section{Materials and methods}

Patient selection and study design

We performed a subgroup study within the HONEUR knee cohort, a prospective observational cohort study in general practice, in which patients were included if they consulted their general practitioner for the first time because of acute or chronic knee complaints. The study design of this general cohort, in which a total of 1068 patients were included, has been described in detail by Heintjes et al. [16]. The subgroup under investigation in the present study consisted of consecutively included patients aged 18 to 65 years who were consulting their general practitioners because of an acute knee trauma within the preceding 5 weeks from March 2002 until October 2003 [17]. Patients were excluded in the case of severe injury requiring immediate hospital referral, if a fracture was demonstrated in those referred for conventional radiography, or if there were contra-indications for MRI. The study was approved by the institutional review board of the Maasstad Ziekenhuis, Rotterdam, The Netherlands, and written informed consent was obtained from each patient.

\section{MRI technique and interpretation}

Magnetic resonance imaging was performed within 3 to 6 weeks of trauma and repeated after approximately 1 year follow-up. For both examinations, we applied the same technique and pulse sequences, using a 1.0 Tesla wholebody MRI unit and a dedicated knee coil. The imaging protocol consisted of sagittal T1-, T2-, and proton densityweighted fast spin-echo sequences, coronal $\mathrm{T} 2 *$-weighted gradient echo and fat-suppressed T2-weighted fast spinecho sequences, and an axial proton density-weighted fast spin-echo sequence.

The initial and follow-up MRI examinations were evaluated for the presence of degenerative abnormalities of the femorotibial joint. For this purpose, we used the items of the Knee Osteoarthritis Scoring System (KOSS) [18]. To grade the severity of OA we used the Kellgren and 
Lawrence scoring system, which was originally developed for grading osteoarthritic change on conventional radiography [19] (Table 1). In addition to degenerative abnormalities including meniscal lesions and bone marrow oedema, we assessed the presence of ligamentous lesions on the initial MRI. Findings were documented on a standardised report form.

Two independent readers (one senior radiology resident (SB) and one experienced musculoskeletal radiologist (DV)) evaluated the initial MRI examination. In the case of discrepancies, consensus was reached through discussion. The follow-up MRI examinations were assessed by three independent readers (two senior radiology residents (EHGO and IMK) and one experienced musculoskeletal radiologist $(\mathrm{JHH})$ ). In the case of discrepancies the majority opinion was used for analysis.

To reflect clinical practice, both the reports and the images of the initial MRI examination were available on follow-up MRI evaluation. In this observational cohort study, the treating general practitioner was not informed of the findings on the initial MRI unless findings required immediate treatment. Hence, the treatment strategy was not influenced by the MRI findings. If a patient was referred to a medical specialist, the initial MRI report was provided on request, so as to avoid unnecessary repetition of an MRI examination.

\section{Self-reported questionnaires}

Self-reported questionnaires were completed at baseline and 12 months after trauma. On the questionnaires, we recorded a pain score measured on an 11-point numeric rating scale ranging from 0 (no pain) to 10 (unbearable pain) [20], the
Lysholm knee function score [21], recurrence, level of sports activities, referral to a medical specialist, and whether an operation had been performed. At 12 months we also scored perceived recovery as rated by the patient and measured on a 7-point Likert scale, ranging from 'completely recovered' to 'worse than ever'. Both the initial and follow-up MRI readers were blinded to the reported scores on the questionnaires.

\section{Data analysis}

Univariate and multivariate binary logistic regression analysis was performed using SPSS 11.0 for Windows. As the binary outcome, we defined any increase on the Kellgren and Lawrence scale on follow-up MRI compared with initial MRI. Therefore, this could either be new degenerative change in a previously unaffected knee or deterioration of pre-existing osteoarthritis.

We analysed the following independent variables: age (both continuous and dichotomised at age 50 years), sex, physique as measured by body weight and body mass index (both continuous and dichotomised at 25 and $30 \mathrm{~kg} / \mathrm{m}^{2}$ ), sports injury as the setting of knee trauma, baseline pain score measured by the 11-point numeric rating scale (continuous and dichotomised at 6 points or higher), Lysholm knee function score (continuous and dichotomised at 50 points). The prognostic value of the following knee lesions on initial MRI was also evaluated: meniscal lesion (degenerative, tear, or combined; lateral and medial meniscus analysed separately or in combination), anterior cruciate ligament rupture (partial, total, or combined), bone marrow oedema in the distal femur or proximal tibia (medial and lateral femorotibial joint compartments analysed separately

Table 1 Cross-tabulation of femorotibial Kellgren and Lawrence score on initial versus follow-up MRI

\begin{tabular}{|c|c|c|c|c|c|c|c|}
\hline & & \multicolumn{5}{|c|}{ Kellgren and Lawrence score on initial MRI } & \multirow[b]{2}{*}{ Tota } \\
\hline & & Grade 0 (normal) & Grade 1 & Grade 2 & Grade 3 & Grade 4 & \\
\hline \multirow{6}{*}{$\begin{array}{l}\text { Kellgren and Lawrence } \\
\text { score on follow-up MRI }\end{array}$} & Grade 0 (normal) & 62 & - & - & - & - & 62 \\
\hline & Grade 1 & 18 & 16 & - & - & - & 34 \\
\hline & Grade 2 & 4 & 1 & 5 & - & - & 10 \\
\hline & Grade 3 & - & 2 & - & 4 & - & 6 \\
\hline & Grade 4 & - & - & - & 2 & 3 & 5 \\
\hline & Total & 84 & 19 & 5 & 6 & 3 & 117 \\
\hline
\end{tabular}

Table presents number of patients

Kellgren and Lawrence Score:

Grade 0: no degenerative abnormalities

Grade 1: minimal osteophyte of unknown significance

Grade 2: osteophytes without joint space narrowing

Grade 3: moderate joint space narrowing

Grade 4: severe joint space narrowing with sclerosis of subchondral bone 
and combined). As there were relatively few total PCL and collateral ligament ruptures, we combined these lesions as a total ACL rupture, resulting in a combined variable indicating any total rupture of either cruciate of the collateral ligament. In the univariate analysis, we regarded variables as statistically significant with a $p$ value of less than 0.10 . In the multivariate analysis, we used a statistically significant threshold of a $p$ value of 0.05 .

We missed data on sports injury in 6 patients. As the association with a sports injury was strongly correlated with age and sex, we used these variables in a logistic regression analysis to impute the missing data on sports injury. Data on baseline pain score and Lysholm knee function score were missing in 3 patients, the pain score and perceived recovery data after 12 months' follow-up were lacking in 7 and 1 patients respectively. We imputed these data with the mean values of the other patients, as it has been demonstrated that imputation of missing data reduces the risk of bias and is preferable over complete case analysis [22, 23].

Sixteen patients underwent surgery during follow-up. As this study was not aimed at therapy, the exact type of surgical or arthroscopic procedures was not known in every operated patient. To explore the possible effect of surgery on the result of the analysis, we performed a sensitivity analysis from which the operated patients were excluded.

Perceived recovery measured on the self-reported questionnaire at 12 months' follow-up was regarded as an indicator of clinical outcome. The expected course of recovery one year after knee trauma was complete recovery or at least strong improvement. Following dichotomisation of this variable into complete recovery or strong improvement versus some improvement, unchanged or deteriorated, we analysed the association between OA progression on the Kellgren and Lawrence score and the clinical outcome using Fisher's exact test.

\section{Results}

One hundred and thirty-four patients were included and underwent MRI at baseline. Seventeen patients were not followed up because they refused participation or because they had moved and we were unable to contact them. Thus, followup MRI was performed in 117 patients (67 male, 50 female; mean age 41 years, standard deviation 12.2 years, range 18-63 years). The mean time between MRI examinations was 403 days (standard deviation 57.8 days, range 315-675 days). A flow diagram indicating the selection of subjects and the flow of patients through the study is presented in Fig. 1.

A cross-tabulation of the Kellgren and Lawrence score on initial versus follow-up MRI is listed in Table 1. At one year follow-up, a total of 27 knees showed an increase on the Kellgren and Lawrence scale; 22 of these were initially

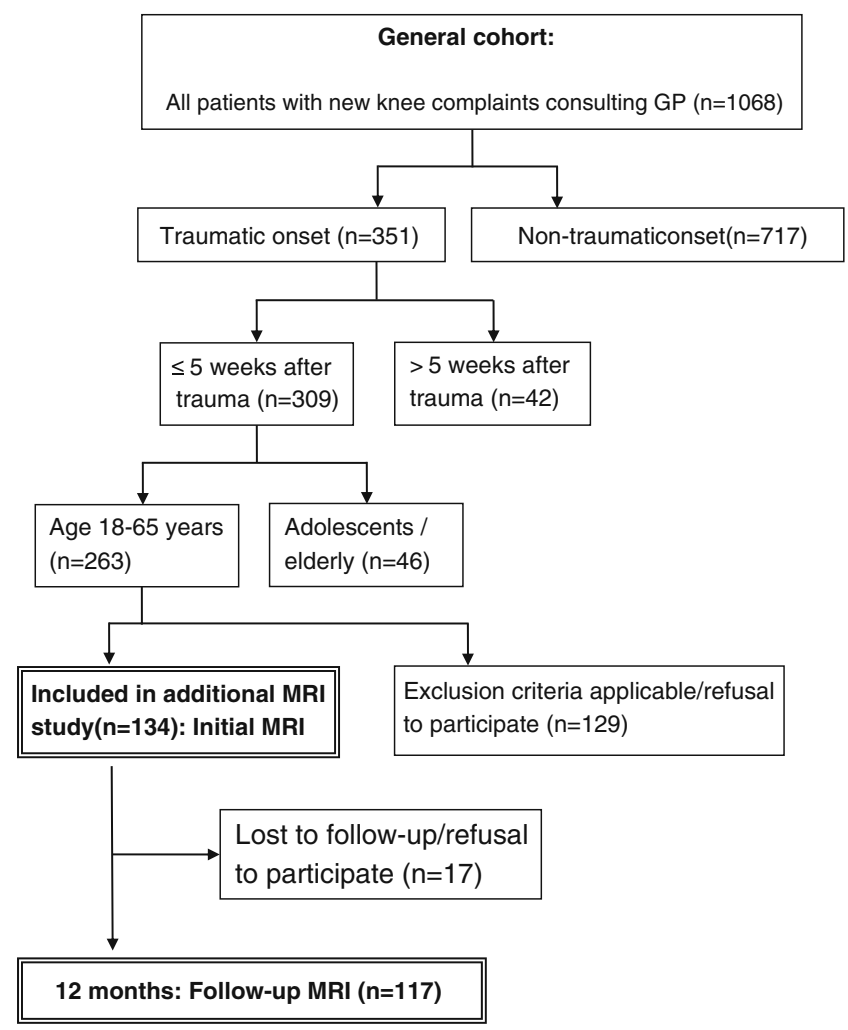

Fig. 1 Flow diagram indicating the selection of subjects and the flow of patients through the study

normal. Five patients demonstrated deterioration of preexisting degenerative change.

The results of the univariate logistic regression analysis are presented in Table 2. Age above 50 years, body mass index greater than $25 \mathrm{~kg} / \mathrm{m}^{2}$, and the presence of bone marrow oedema on initial MRI (both for medial and lateral compartments separately and combined) were significant predictors of increased Kellgren and Lawrence score after one year follow-up. We included age above 50 years, body mass index greater than $25 \mathrm{~kg} / \mathrm{m}^{2}$, and bone marrow oedema (medial and lateral compartments combined) in the multivariate logistic regression analysis. Although age and body mass index both were no longer statistically significant when controlling for bone marrow oedema (OR 1.51 (95\% CI $0.56-4.12$ ), $p=0.42$ and OR 2.07 (95\% CI $0.75-5.73$ ), $p=0.16$ respectively), we kept these variables in the model, as these are established risk factors for osteoarthritis. Thus, in the final multivariate model medial and/or lateral femorotibial bone marrow oedema on initial MRI was the only statistically significant predictor of increased Kellgren and Lawrence score on follow-up MRI after one year (OR 5.29 (95\% CI 1.64-17.1), $p=0.005$ ).

In the sensitivity analysis of 101 conservatively treated patients excluding the 16 patients who had undergone surgery during follow-up, we identified the same statistically significant variables in univariate logistic regression analysis 
Table 2 Results of the univariate logistic regression analysis

\begin{tabular}{|c|c|c|c|}
\hline Covariate & $\begin{array}{l}\text { Frequency in study population } \\
\text { of } 117 \text { patients (percentage) }{ }^{a}\end{array}$ & $\begin{array}{l}\text { Odds ratio }(95 \% \\
\text { confidence interval) }\end{array}$ & $p$ value \\
\hline Age (continuous) & 41.0 years $(12.2,18-63)^{\mathrm{a}}$ & $1.03 / \mathrm{yr}(0.99-1.07)$ & 0.10 \\
\hline Age $>50$ years & $30(26)$ & $2.57(1.02-6.44)$ & 0.04 \\
\hline Male sex & $67(57)$ & $0.74(0.30-1.78)$ & 0.50 \\
\hline Body weight & $82.6 \mathrm{~kg}(15.6,40-129)^{\mathrm{a}}$ & $1.01 / \mathrm{kg}(0.98-1.04)$ & 0.41 \\
\hline Body mass index (continuous) & $26.3 \mathrm{~kg} / \mathrm{m} 2(4.1,17-40)^{\mathrm{a}}$ & $1.07(0.97-1.19)$ & 0.18 \\
\hline Body mass index $>25 \mathrm{~kg} / \mathrm{m}^{2}$ & $69(59)$ & $2.39(0.92-6.21)$ & 0.07 \\
\hline Body mass index $>30 \mathrm{~kg} / \mathrm{m}^{2}$ & $20(17)$ & $1.55(0.53-4.53)$ & 0.42 \\
\hline Sports injury ${ }^{\mathrm{b}}$ & $59(50)$ & $1.08(0.46-2.55)$ & 0.87 \\
\hline Baseline pain score continuous ${ }^{c}$ & $4.6(2.3,0-10)^{\mathrm{a}}$ & $1.01(0.84-1.22)$ & 0.92 \\
\hline Baseline pain score $>6$ points $^{c}$ & $44(38)$ & $0.79(0.32-1.94)$ & 0.60 \\
\hline Baseline Lysholm score continuous ${ }^{\mathrm{c}}$ & $65.1(18.8,16-100)^{\mathrm{a}}$ & $0.99(0.97-1.01)$ & 0.25 \\
\hline Baseline Lysholm score $>50^{\mathrm{c}}$ & $88(75)$ & $0.72(0.28-1.89)$ & 0.51 \\
\hline \multicolumn{4}{|l|}{ Lesions on initial MRI } \\
\hline Total ACL rupture & $12(10)$ & $1.13(0.28-4.49)$ & 0.87 \\
\hline Total or partial ACL rupture & $21(18)$ & $1.90(0.68-5.33)$ & 0.22 \\
\hline Total cruciate or collateral ligament rupture & $14(12)$ & $2.05(0.62-6.73)$ & 0.24 \\
\hline Lateral meniscal tear & $24(21)$ & $0.85(0.28-2.54)$ & 0.77 \\
\hline Lateral meniscus degenerative lesion or tear & $47(40)$ & $0.84(0.35-2.05)$ & 0.71 \\
\hline Medial meniscal tear & $32(27)$ & $1.82(0.73-4.55)$ & 0.20 \\
\hline Medial meniscus degenerative lesion or tear & $91(78)$ & $1.34(0.45-3.97)$ & 0.60 \\
\hline Lateral or medial meniscal tear & $43(37)$ & $1.52(0.64-3.65)$ & 0.35 \\
\hline Lateral or medial meniscus degenerative lesion or tear & $95(81)$ & $1.03(0.34-3.09)$ & 0.97 \\
\hline Bone marrow oedema in lateral compartment & $45(39)$ & $3.06(1.26-7.42)$ & 0.01 \\
\hline Bone marrow oedema in medial compartment & $53(45)$ & $3.14(1.27-7.77)$ & 0.01 \\
\hline Bone marrow oedema in lateral or medial compartment & $67(57)$ & $6.01(1.92-18.8)$ & 0.002 \\
\hline
\end{tabular}

${ }^{\mathrm{a}}$ For continuous variables, we report mean (standard deviation, range)

${ }^{\mathrm{b}} 6$ missing values were imputed

${ }^{\mathrm{c}} 3$ missing values were imputed

(results not tabulated). Similarly, femorotibial bone marrow oedema was the only statistically significant variable in multivariate analysis (OR 5.38 (95\% CI 1.67-17.3), $p=0.005$ ).

A cross-tabulation of perceived recovery versus stable or progressive degenerative change on follow-up MRI is shown in Table 3. Ninety-seven patients reported complete recovery or strong improvement after 12 months' followup, whereas 3 patients experienced some deterioration. Seventeen of the 27 patients with new or progressive osteoarthritic change reported complete recovery or strong improvement in terms of perceived recovery versus 80 of

Table 3 Perceived recovery after 12 months and progression of degenerative change on follow-up MRI

\begin{tabular}{llllll}
\hline & \multicolumn{2}{l}{ Perceived recovery } & & \\
\cline { 2 - 6 } & $\begin{array}{l}\text { Complete } \\
\text { recovery }\end{array}$ & $\begin{array}{l}\text { Strong } \\
\text { improvement }\end{array}$ & $\begin{array}{l}\text { Some } \\
\text { improvement }\end{array}$ & $\begin{array}{l}\text { Unchanged } \\
\text { Total }\end{array}$ & $\begin{array}{l}\text { Some } \\
\text { deterioration }\end{array}$ \\
\hline Absent or unchanged pre-existing degenerative change & 23 & 57 & 6 & 3 & 1 \\
New or progressive degenerative change & 7 & 10 & 7 & 1 & 2 \\
Total & 30 & 67 & 13 & 4 & 3 \\
\hline
\end{tabular}

No patients selected the categories strong deterioration or worse than ever

Table displays number of patients 
the 90 patients with absent or unchanged OA. There was a statistically significant association between new or progressive osteoarthritic change on follow-up MRI and perceived recovery (Fisher's exact test $\mathrm{p}$ value 0.003 ).

\section{Discussion}

We evaluated prognostic factors, including initial MRI findings after knee trauma, for new onset or progression of OA of the femorotibial joint, as assessed by follow-up MRI after one year. Unlike previously published studies on this subject, we conducted this study in the general practice population as most patients with traumatic knee injury are managed in a primary care setting in our country. In the univariate logistic regression analysis, higher age ( $>50$ years), higher body mass index $(>25 \mathrm{~kg} / \mathrm{m} 2)$, and bone marrow oedema were associated with new or progressive osteoarthritic changes of the femorotibial joint one year after trauma. In multivariate logistic regression analysis, however, only bone marrow oedema was statistically significant and it was found to be a strong predictor. Whereas previously published studies focused on the longterm ( 2 years and more) risk of developing OA after knee injury, we focused on the risk of developing MRI signs of OA during the first year following trauma [7, 8, 24].

Both higher age and higher body mass index were identified as risk factors for progression of knee osteoarthritis in previous case control studies and population based studies [2, 4, 25]. Although we only found this association in univariate logistic regression analysis, we kept these variables in the model, as these are established risk factors for osteoarthritis. It is possible that the number of patients was too small to demonstrate significance. Apparently bone marrow oedema is more important in the onset or progression of knee osteoarthritis than the other two risk factors.

Our findings are consistent with other reports on bone marrow oedema. In the Pond-Nuki dog model of OA, bone marrow lesions were considered a very early sign of OA [10]. In a longitudinal study by Felson et al. bone marrow oedema was suggested to be a powerful predictor of disease progression in non-traumatically injured patients with symptomatic OA of the knee [9]. Kubota et al. recently found a relationship between bone marrow abnormalities and the progression of knee OA [26].

Histopathologically, bone marrow oedema after traumatic knee injury (also referred to as bone bruise) is thought to be the result of microfractures and haemorrhage in the subcortical trabecular bone. Bone bruise-related cartilage changes are reported to be the result of chondrocyte degeneration with proteoglycan loss [27]. As damage to the articular cartilage is thought to be an important factor in the development of OA, this may explain the relationship between traumatic bone marrow oedema and early progression of OA.

In a previous study, we found that the median healing time of post-traumatic bone bruises in our study group was 42.1 weeks [28]. The present study suggests that although the bone bruise itself is healed within a year, a long-term effect may be initiated by this lesion, i.e. the development of osteoarthritic change of the knee joint.

Our study has several limitations. We only evaluated the femorotibial joint because there were too few patients $(n=4)$ with new onset or progressive patellofemoral OA to justify a separate logistic regression analysis. We considered that a pooled analysis of these joints would be meaningless, because the pathogenesis of femorotibial and patellofemoral $\mathrm{OA}$ and the effect of trauma is probably different. In recent MRI-based studies on OA, the Whole Organ Magnetic Resonance Imaging Score (WORMS) and Knee Osteoarthritis Scoring System (KOSS) have been used to evaluate the severity of degenerative change $[15,18]$. At the time of the baseline MRI performed in our study (2002-2003), these semi-quantitative scoring systems had not been introduced, although the items scored were almost identical to KOSS. For reasons of comparability and to avoid interfering with the prospective study design, we used the KOSS items for the evaluation of progression of OA, but we continued using the adapted Kellgren and Lawrence system for grading OA on the 1-year follow-up MRI [19]. The outcome was defined as any increase on the Kellgren and Lawrence scale. We acknowledge that this refers to a heterogeneous group of patients including those with new onset OA and those with progression of pre-existing osteoarthritic changes. Most patients with progression of OA on MRI, however, had no OA initially, indicating that the results of this study were largely based on patients who developed new onset degenerative change within one year of trauma. Most of these patients developed mild degenerative change of Kellgren and Lawrence grade 1-2 severity. As the contralateral knee was not imaged, we cannot exclude that degeneration as part of the natural history explains part of our findings. Furthermore, which proportion of osteoarthritic change is attributable to trauma is unknown, but whether or not the OA is attributable to the trauma, although interesting pathophysiologically, is clinically less relevant. The important point is that in a patient with knee injury, bone marrow oedema predicts the development of early OA.

We performed our study in the primary care setting, and consequently patients with more severe traumatic knee injuries were not included because they were referred to secondary care immediately. This may explain the relatively limited number of meniscal injuries and cruciate ligament injuries in our patient population, which implied a limited power to demonstrate a statistically significant association between these lesions and early progression of OA. The 
true extent to which meniscal damage and cruciate ligament injuries predict early progression of OA may be underestimated in our study, as both types of lesions have been reported to be risk factors for progression of OA [6-8].

As it was not the aim of the study to evaluate treatment for traumatic knee lesions, we did not record in detail what procedure was performed in the operated patients. To explore the consequences of this limitation, we performed a sensitivity analysis excluding the operated patients. The same prognostic factor with a similar odds ratio was found, indicating that the results of the analysis were not influenced by the type of treatment, underscoring the robustness of the model.

We found a significant association between new onset or progression of femorotibial OA at follow-up MRI and clinical outcome as measured by perceived recovery. All patients in our study sustained a knee injury resulting in acute trauma-related symptoms. One year after trauma the expected natural healing course would be complete recovery or at least strong improvement. As the disabling symptoms in the initial stage after trauma are no longer present one year after trauma, a perceived recovery less than "strong improvement" in all likelihood reflects the effect of new onset or progressive OA. In light of our results, this stresses the importance of identifying those patients with bone bruise in the initial stage after trauma and those with no or minor improvement of clinical outcome after trauma, as these patients are at high risk of developing new onset OA. In the future these patients may benefit from the development of treatment strategies targeting bone bruises.

In conclusion, the results of this study demonstrate that bone marrow oedema on initial MRI after knee trauma is a strong predictor, and in multivariable analysis the only predictor, of new onset and progression of knee osteoarthritis on 1-year follow-up MRI, which is reflected in the clinical outcome.

Acknowledgements We thank Ms Wibeke van Leeuwen and Mrs Caroline van Bavel for their assistance with the data management.

The results of this study have been accepted for scientific poster presentation at the ECR Congress 2009 in Vienna, Austria

Funding This study was funded in part by College voor Zorgverzekeringen (Dutch Health Insurance Board).

Open Access This article is distributed under the terms of the Creative Commons Attribution Noncommercial License which permits any noncommercial use, distribution, and reproduction in any medium, provided the original author(s) and source are credited.

\section{References}

1. Peterfy CG (2002) Imaging of the disease process. Curr Opin Rheumatol 14:590-596
2. Spector TD, Hart DJ, Doyle DV (1994) Incidence and progression of osteoarthritis in women with unilateral knee disease in the general population: the effect of obesity. Ann Rheum Dis 53:565-568

3. Roos EM (2005) Joint injury causes knee osteoarthritis in young adults. Curr Opin Rheumatol 17:195-200

4. Reijman M, Pols HA, Bergink AP et al (2007) Body mass index associated with onset and progression of osteoarthritis of the knee but not of the hip: the Rotterdam Study. Ann Rheum Dis 66:158-162

5. Van der Linden MW, Westert GP, de Bakker DH, Schellevis FG (2004) Tweede nationale studie naar ziekten en verrichtingen in de huisartspraktijk: klachten en aandoeningen in de bevolking en in de huisartspraktijk. Nivel, Rijksinstituut voor volksgezondheid en milieu, Utrecht, Bilthoven

6. Berthiaume MJ, Raynauld JP, Martel-Pelletier J et al (2005) Meniscal tear and extrusion are strongly associated with progression of symptomatic knee osteoarthritis as assessed by quantitative magnetic resonance imaging. Ann Rheum Dis 64:556-563

7. Sharma L, Eckstein F, Song J et al (2008) Relationship of meniscal damage, meniscal extrusion, malalignment, and joint laxity to subsequent cartilage loss in osteoarthritic knees. Arthritis Rheum 58:1716-1726

8. Lohmander LS, Englund PM, Dahl LL, Roos EM (2007) The long-term consequence of anterior cruciate ligament and meniscus injuries: osteoarthritis. Am J Sports Med 35:1756-1769

9. Felson DT, McLaughlin S, Goggins J et al (2003) Bone marrow edema and its relation to progression of knee osteoarthritis. Ann Intern Med 139:330-336

10. Libicher M, Ivancic M, Hoffmann M, Wenz W (2005) Early changes in experimental osteoarthritis using the Pond-Nuki dog model: technical procedure and initial results of in vivo MR imaging. Eur Radiol 15:390-394

11. Spector TD, Hart DJ, Huskisson EC (1991) The use of radiographs in assessing the severity of knee osteoarthritis. J Rheumatol Suppl 27:38-39

12. Lawrence JS, Bremner JM, Bier F (1966) Osteo-arthrosis. Prevalence in the population and relationship between symptoms and x-ray changes. Ann Rheum Dis 25:1-24

13. Kijowski R, Blankenbaker DG, Stanton PT, Fine JP, De Smet AA (2006) Radiographic findings of osteoarthritis versus arthroscopic findings of articular cartilage degeneration in the tibiofemoral joint. Radiology 239:818-824

14. Link TM, Steinbach LS, Ghosh S et al (2003) Osteoarthritis: MR imaging findings in different stages of disease and correlation with clinical findings. Radiology 226:373-381

15. Peterfy CG, Guermazi A, Zaim S et al (2004) Whole-Organ Magnetic Resonance Imaging Score (WORMS) of the knee in osteoarthritis. Osteoarthritis Cartilage 12:177-190

16. Heintjes EM, Berger MY, Koes BW, Bierma-Zeinstra SM (2005) Knee disorders in primary care: design and patient selection of the HONEUR knee cohort. BMC Musculoskelet Disord 6:45

17. Boks SS, Vroegindeweij D, Koes BW, Hunink MM, Bierma-Zeinstra SM (2006) Magnetic resonance imaging abnormalities in symptomatic and contralateral knees: prevalence and associations with traumatic history in general practice. Am J Sports Med 34:1984-1991

18. Kornaat PR, Ceulemans RY, Kroon HM et al (2005) MRI assessment of knee osteoarthritis: Knee Osteoarthritis Scoring System (KOSS)inter-observer and intra-observer reproducibility of a compartmentbased scoring system. Skeletal Radiol 34:95-102

19. Petersson IF, Boegard T, Saxne T, Silman AJ, Svensson B (1997) Radiographic osteoarthritis of the knee classified by the Ahlback and Kellgren \& Lawrence systems for the tibiofemoral joint in people aged 35-54 years with chronic knee pain. Ann Rheum Dis $56: 493-496$

20. Herr KA, Spratt K, Mobily PR, Richardson G (2004) Pain intensity assessment in older adults: use of experimental pain to 
compare psychometric properties and usability of selected pain scales with younger adults. Clin J Pain 20:207-219

21. Briggs KK, Kocher MS, Rodkey WG, Steadman JR (2006) Reliability, validity, and responsiveness of the Lysholm knee score and Tegner activity scale for patients with meniscal injury of the knee. J Bone Joint Surg Am 88:698-705

22. Van der Heijden GJ, Donders AR, Stijnen T, Moons KG (2006) Imputation of missing values is superior to complete case analysis and the missing-indicator method in multivariable diagnostic research: a clinical example. J Clin Epidemiol 59:1102-1109

23. Schafer J (1997) Analysis of incomplete multivariate data. Chapman \& Hall, London

24. Cooper C, Snow S, McAlindon TE et al (2000) Risk factors for the incidence and progression of radiographic knee osteoarthritis. Arthritis Rheum 43:995-1000
25. Hochberg MC, Lethbridge-Cejku M, Scott WW Jr, Reichle R, Plato CC, Tobin JD (1995) The association of body weight, body fatness and body fat distribution with osteoarthritis of the knee: data from the Baltimore Longitudinal Study of Aging. J Rheumatol 22:488-493

26. Kubota M, Ishijima M, Kurosawa $\mathrm{H}$ et al (2010) A longitudinal study of the relationship between the status of bone marrow abnormalities and progression of knee osteoarthritis. J Orthop Sci 15:641-646

27. Thiryayi WA, Thiryayi SA, Freemont AJ (2008) Histopathological perspective on bone marrow oedema, reactive bone change and haemorrhage. Eur J Radiol 67:62-67

28. Boks SS, Vroegindeweij D, Koes BW, Bernsen RM, Hunink MG, Bierma-Zeinstra SM (2007) MRI follow-up of posttraumatic bone bruises of the knee in general practice. AJR Am J Roentgenol 189:556-562 